\title{
Mode Transitions for the ST7 Disturbance Reduction System Experiment
}

\author{
O. C. Hsu, P. G. Maghami, F. L. Markley ${ }^{\dagger}$, and J. R. O'Donnell Jr. ${ }^{\ddagger}$ \\ NASA Goddard Space Flight Center \\ Mission Engineering and Systems Analysis Division \\ Greenbelt, MD 20771
}

\begin{abstract}
The Space Technology 7 Disturbance Reduction System experiment will perform an onorbit system-level validation of two technologies: a gravitational reference sensor employing a free-floating test mass and a set of colloidal micronewton thrusters. The Disturbance Reduction System is designed to maintain the spacecraft's position with respect to a freenoating test mass to less than $10 \mathrm{~nm} / \sqrt{\mathrm{Hz}}$ over the frequency range of 1 to $30 \mathrm{mHz}$ This paper presents the modes that compose the Disturbance Reduction System spacecraft control as well as the strategy used to transition between modes. A high-fidelity model of the system, which incorporates rigid-body models of the spacecraft and two test masses (18 degrees of freedom), is developed and used to evaluate the performance of each mode and the eficicacy of the transition strategy.
\end{abstract}

\section{Introduction}

$\mathrm{T}$

HE Space Technology 7 (ST7) mission is a Disturbance Reduction System (DRS) flight validation experiment within NASA's New Millennium Program.' New Millennium Program missions are intended to validate advanced technologies that have not flown in space in order to reduce the risk of their infusion into future NASA science missions. The ST7-DRS incorporates two technologies: a highly sensitive Gravitational Reference Sensor (GRS), to measure the position and attitude of a spacecraft with respect to an internal free-floating test mass, and a set of colloidal micronewton thrusters (CMNT) to provide low-noise control of the spacecraft for drag-free flight. The DRS is scheduled to fly on the European Space Agency's (ESA) LISA Pathfinder (LPF) spacecraft in 2008, (LISA Pathfinder is an ESA mission funded by the ESA member states and NASA) and will operate in an orbit about the Earth-Sun $L_{1}$ point. The DRS is designed to maintain the spacecraft's position, with respect to the GRS free-floating test mass, to less than $10 \mathrm{~nm} / \sqrt{\mathrm{Hz}}$, over DRS's science band, a frequency range from 1 to $30 \mathrm{mHz}$. This requirement will help ensure that the residual accelerations on the test masses (beyond gravitational acceleration) will be below the DRS goal of $3 \times 10^{-14}\left[1+(f / 3 \mathrm{mHz})^{2}\right] \mathrm{m} / \mathrm{s}^{2} / \sqrt{\mathrm{Hz}}$. The DRS instrument package consists of two gravitational reference sensors, two sets of four colloidal micronewton thrusters each for position and attitude control, a pair of interferometers to measure the distance of each test mass with respect to the spacecraft in the direction of the separation between the test masses, and associated electronics.

This paper describes the design of the five primary control modes of the spacecraft Dynamic Control System (DCS) of the DRS and discusses the strategy for transitioning from one mode to another. A high-fidelity (HiFi) Simulink ${ }^{\mathrm{TM}}$ model of the system, which includes the dynamics of the spacecraft and the two test masses, is developed. It includes actuation and measurement noise as well as the major disturbance sources acting on the spacecraft and test masses. This model is used to verify the performance of each of the control modes as well as to ensure that the proposed mode transition strategy is feasible.

\section{Control Modes}

Five primary DCS control modes have been designed for ST7-DRS spacecraft control. These five operational modes are attitude-only (AO), accelerometer (AC), initial drag-free (DF1), interim drag-free (DF2), and full dragfree or science mode (SM). The AO mode of the DCS controls the spacecraft attitude based on measurements from

- Aerospace Engineer, Flight Dynamics Analysis Branch, Code 595, Member AIAA

${ }^{\dagger}$ Associate Branch Head, Flight Dynamics Analysis Branch, Code 595, Senior Member AIAA

${ }^{\ddagger}$ Systems Engineer, Guidance, Navigation, and Control Systems Engineering Branch, Code 591, AIAA Fellow 
the star tracker (ST) to ensure that the attitude error remains within prescribed limits. The AC mode incorporates the acceleration data from one of the GRS units and uses this information along with the star tracker data to maintain the spacecraft attitude and to null the accelerations at the Reference Test Mass (RTM) of the GRS unit. The DF1 mode incorporates position data from the reference GRS unit-three-axis measurements of the gap between the test mass and its reference housing-and star tracker data to null any differences between the RTM and its desired position and maintain the spacecraft attitude. The DF2 mode begins to incorporate acceleration data of the Non-Reference Test Mass (NTM) from the other GRS unit, along with the position data for the RTM and star tracker data to null the accelerations on the NTM, to position the spacecraft such that GRS Unit is in the desired position with respect to the RTM, and to maintain the spacecraft attitude. The final mode, SM mode, incorporates position data from both test masses and the star tracker data to reduce any errors in the position of the spacecraft with respect to the RTM location in all translational directions, to reduce errors in the spacecraft position with respect to the NTM in the translational directions transverse to the distance measurement, and to maintain the spacecraft attitude.

The GRS control modes are coupled with the DCS modes, and the primary modes for the GRS are caged, accelerometer, drag-free, and suspended drag-free mode. In the caged mode, the GRS prevents any relative motion of the test mass with respect to the GRS housing. In the accelerometer mode, the GRS applies large forces and torques on the test mass to limit the relative motion of the test mass with respect to its housing while sensing the linear and angular acceleration acting upon the test mass. In drag-free mode, the GRS controller is limited to attitude control of the test mass. The drag-free mode is designed to maintain the attitude of the test mass by applying torques to the test mass at low frequencies while also measuring the position of the test mass with respect to the GRS housing. The suspended drag-free mode is similar to the drag-free mode with the additional capability of providing translational correction of the test mass below the DRS measurement frequency band.

This paper is limited to the attitude control system (ACS) and the drag-free control system (DFCS) that are components of the five DCS control modes. However, as suspension loops for the two GRS units are also required for the analysis and implementation of the overall system, preliminary controllers for the suspension loops are included in the analyses and results presented in the paper.

\section{A. Attitude-Only Mode}

Attitude-Only (AO) mode is the first mode in the DCS control strategy and the purpose of this control mode is to reduce any transients during hand-over from LPF control to DRS control, to maintain the spacecraft attitude within a specified attitude dead-band, and orient the spacecraft at a prescribed attitude, nominally sun-pointing. Therefore, the ACS is the only operational control system on the spacecraft side. The GRSs are in either caged or accelerometer modes. The attitude



Figure 1. AO Mode Controller Dependencies control loop comprises three single-input/single-output (SISO) controllers which use the attitude errors and derived rates obtained from ST measurements to compute the required spacecraft torque commands. A diagram of the AO mode controller and its dependency is shown in Figure 1. The controller is given by the equation:

$$
\vec{T}(z)=K_{a l}(z) \vec{\theta}(z)
$$

Here, controller $K_{a l}(z)$ denotes the low-bandwidth attitude control designed to maintain the coarse pointing requirements of the spacecraft and the output $\vec{T}$ is the commanded spacecraft torque. It is a SISO controller with each loop comprising a PID controller with an appropriate digital attenuation filter.

\section{B. Accelerometer Mode}

Accelerometer (AC) mode is the second mode in the DCS control strategy and the purpose of this mode is to facilitate the transition into the initial drag-free mode by reducing the acceleration levels on the reference test mass. AC comprises two control systems, ACS and DFCS. In this mode, the reference GRS is assumed to be in accelerometer mode and the non-reference GRS is assumed either to be caged or to be in accelerometer mode. The ACS is the same as the one used in the $\mathrm{AO}$ mode.

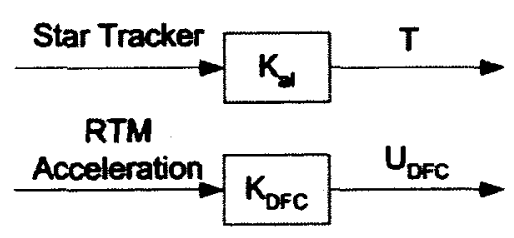

Figure 2. AC Mode Controller Dependencies 
The DFCS uses the accelerometer signals from the reference GRS to control the position of the spacecraft in order to reduce the translational acceleration levels of the reference test mass, where either test mass can be chosen as the reference test mass. A diagram of the AC mode controllers and their dependencies are shown in Figure 2 . The controller is given by:

$$
\vec{u}_{D F C}(z)=A_{g r s s c} K_{D F C}(z) \vec{r}_{R T M}(z)
$$

Where $A_{\text {grs } 2 x}$ denotes the coordinate transformation matrix from the GRS frame to the spacecraft body frame, $K_{D F C}(z)$ represents a combined controller/filter, the vector $\overrightarrow{\vec{r}}_{R T M}$ represents the GRS acceleration measurement vector of the RTM in the accelerometer mode, and the output $\vec{u}_{D F C}$ is the commanded spacecraft force.

\section{Initial Drag-Free Mode}

The third DCS mode is the initial drag-free (DF1) mode and the purpose of this mode is to establish drag-free control with respect to the reference test mass. The DCS controller comprises two control systems in the DFl mode, the ACS and DFCS systems. The ACS is the same system used in both $\mathrm{AO}$ and $\mathrm{AC}$ mode and has the same responsibility of maintaining the spacecraft's attitude towards the target and ensuring the spacecraft attitude dead-band is not violated. The DFCS controls the position of the spacecraft to establish free-fall motion of the reference test mass. Feed-forward compensation is implemented in the drag-free loop to reduce coupling between the attitude and translation control. A diagram of the DF1 mode controllers and their dependencies are shown



RTM

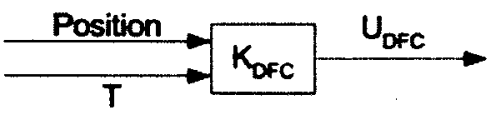

Figure 3. DF1 Mode Controller Dependencies in Figure 3. The controller is given by:

$$
\vec{u}_{D F C}(z)=K_{D F C}(z) A_{g r s s c} \vec{r}_{R T M}(z)+\vec{p}_{R T M} \times\left[H^{*} \vec{T}(z)\right]
$$

Where $A_{g r s 2 s}$ denotes the coordinate transformation matrix from the GRS frame to the spacecraft body frame, $K_{D F C}(z)$ represents a combined controller/filter for the DFCS, the vector $\vec{r}_{R T M}$ represents the GRS position measurement error vector of the RTM with respect to its desired position, the vector $\vec{p}_{R T M}$ represents the nominal position of the RTM relative to the system center of mass, $H$ denotes the product of the spacecraft mass and inverse inertia matrix, and the output $\vec{u}_{D F C}$ is the commanded spacecraft force.

\section{Interim Drag-Free Mode}

The interim drag-free (DF2) mode is identical to DF1, except that the acceleration signals from the non-reference GRS unit are used as part of the attitude control system to reduce the relative motion of the spacecraft about the NTM, thereby allowing for a timely transition into the science mode. The DCS controller comprises two control systems in DF2, the ACS and DFCS. The ACS maintains a coarse attitude with respect to a prescribed target, while reducing the relative motion of the spacecraft about the non-reference test mass in the transverse directions. The ACS is a 5-input/3-output controller,



Figure 4. DF2 Mode Controller Dependencies which uses the three attitude errors (obtained from ST measurement) and the translational acceleration measurements of the non-reference GRS unit in the two directions transverse to the sensitive axis (the line joining the two test masses) to compute the required spacecraft torque commands. A diagram of the DF2 mode controllers and their dependencies are shown in Figure 4. The ACS controller is given by: 


$$
\vec{T}(z)=K_{a l}(z) \vec{\theta}(z)+K_{a h}(z)\left\{\begin{array}{l}
\ddot{y}_{N T M}(z) \\
\ddot{z}_{N T M}(z)
\end{array}\right\}
$$

Here, controller $K_{a l}(z)$ denotes the low-bandwidth part of the attitude control designed to maintain the attitude of the spacecraft. It is a SISO controller with each loop employing a PID controller with an appropriate digital attenuation filter. Controller $K_{a h}(z)$, the complementary attitude controller, represents the part of the attitude controller responsible for centering the spacecraft about the NTM in the transverse directions. The acceleration measurements of the NTM, in the transverse directions $\left(\ddot{y}_{N T M}, \ddot{z}_{N T M}\right)$ from the GRS unit, are computed from the commanded electrostatic forces (while the GRS unit is in accelerometer mode), and are used to compute the required rotational (in GRS frame) acceleration of the GRS package about the drag-free reference test mass. The required rotational acceleration command is then transformed into the spacecraft frame and is used as an input to a SISO controller to regulate the spacecraft attitude in the science band. In order to obtain a pure rotation about the first test mass, feed-forward translation commands are generated and issued to the drag-free controller.

The drag-free controller in DF2 is the same controller used in DF1 and it is designed to control the spacecraft about the RTM in all translational directions, thereby establishing free-fall motion of the RTM.

\section{E. Science Mode}

The Science mode is the nominal mode of the DRS. It is the only mode wherein full drag-free motion is established for both test masses in the measurement band. The DCS control comprises the ACS and DFCS. The attitude control system maintains a coarse attitude with respect to a prescribed target as well as ensures drag-free motion of the non-reference test mass in the transverse directions in the DRS science band. The attitude control loop is a 7-input/3-output controller, which uses the three attitude errors (obtained from ST measurement) and the relative position errors of the test masses in the transverse directions to compute the required spacecraft torque commands. A diagram of the SM mode controllers and their dependencies

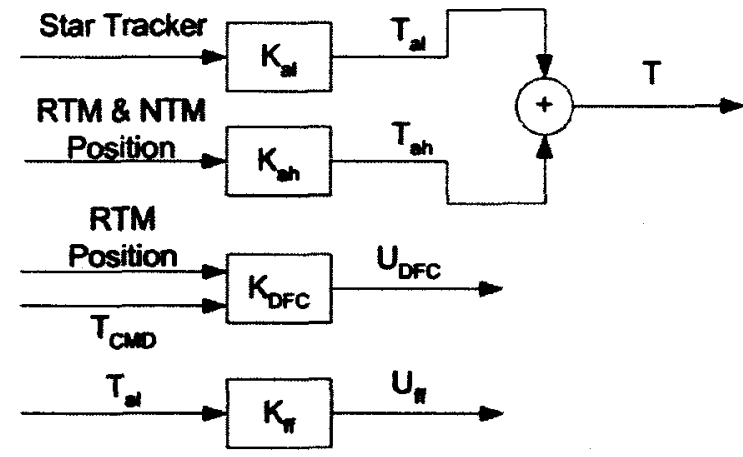

Figure 5. SM Mode Controller Dependencies are shown in Figure 5. The controller is given by:

$$
T(z)=K_{a l}(z) \vec{\theta}(z)+K_{a h}(z)\left\{\begin{array}{l}
y_{R T M}(z) \\
z_{R T M}(z) \\
y_{N T M}(z) \\
z_{N T M}(z)
\end{array}\right\}
$$

Here, controller $K_{a l}(z)$ denotes the low-bandwidth part of the attitude control designed to maintain the attitude of the spacecraft. It is a SISO controller with each loop employing a PID controller with an appropriate digital attenuation filter. Controller $K_{\text {oh }}(z)$, the complementary attitude controller, represents the part of the attitude controller responsible for centering the spacecraft about the non-reference test mass in the transverse directions to the sensitive axis within the science band. The relative position error of the test masses in the transverse direction are used to compute the required rotation (in the GRS frame) of the GRS package about the reference test mass. The required rotation command is then transformed into spacecraft frame and is used as an input to a SISO controller to regulate the spacecraft attitude in the science band. In order to obtain a pure rotation about the first test mass, feedforward translation commands are generated and issued to the drag-free controller. Controller $K_{a h}(z)$ is also designed based on the classical approach, and is a series combination of a lead-lag filter, a PD filter, and a roll-off filter, resulting in a $7^{\text {th }}$-order controller.

The drag-free controller in SM is the same controller used in DF1 and it is designed to control the spacecraft about the RTM in all translational directions, thereby establishing free-fall motion of the RTM. 
In order to make the design more amenable to decentralized control, the low frequency commands generated by controller $K_{a l}(z)$ in the ACS are processed through the proper transformation and are fed as feed-forward commands, in the transverse directions, to the electrostatic suspension loop of the non-reference GRS unit. The feed-forward commands are computed as follows.

$$
u_{f f}(z)=\alpha A_{s c 2 g r s}\left(\vec{p}_{R T M}-\vec{p}_{N T M}\right) \times H_{l} * \vec{T}_{l}(z)
$$

Where $\alpha$ denotes a gain matrix, $A_{s c 2 g r s}$ denotes the coordinate transformation matrix from spacecraft body frame to the GRS frame, the vectors $\vec{p}_{R T M}$ and $\vec{p}_{N T M}$ represent the nominal position of the RTM and NTM relative to the system center of mass, respectively, and $H_{l}$ denotes the product of the mass of the test mass and inverse spacecraft inertia matrix, and $\vec{T}_{l}$ is the commanded torque from the low-bandwidth attitude controller. References 2,3 , and 4 provide a more thorough description of the design and performance of the SM mode.

\section{MODE TRANSITIONS}

The proposed strategy for transitioning between modes is based on direct handover between the control modes with control force/torque mean carryover to minimize initial transients. In this scheme, the mean value of all control signals is computed by using a running-average filter. At the mode transitions, the mean value calculated up to that point is latched/held for the duration of the subsequent control mode and added into the control signal. This procedure occurs differently for control force and control torque. The control force is latched at every mode transition because the DFCS controller changes between each mode and the control states are initialized. The control torque mean is not latched until the DCS transitions from DF1 to DF2 because the previous three modes use the same ACS controllers and the states are not reinitialized between modes. The mode transition strategy is depicted graphically shown in Figure 6.

The main contribution to the mean levels is expected to be the solar pressure force and torque. For the most

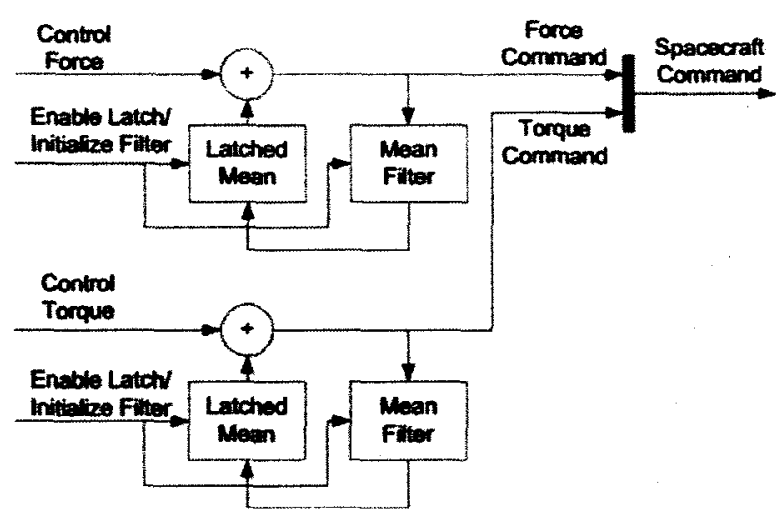

Figure 6. Mode Transition Method part, the transitions between the modes are expected to be sequential, starting from the AO (fighting solar pressure torque) all the way to SM (fighting both solar pressure torque and force). However, possibilities of skipping one or more transitional modes are also being investigated. For example, it may be possible to transition from $\mathrm{AO}$ to $\mathrm{DF} 1$ without having to go through AC mode, or DF1 to SM without having to go through DF2 mode. The decision on the proper time to transition between the modes will be based on expected time-constants of the control loops in each mode as well as the levels of attitude and/or test mass position errors.

\section{Verification Model}

An 18 degree-of-freedom high-fidelity (HiFi) model, which captures the essential dynamics of the ST7-DRS package, has been developed to verify each of the control modes as well as the transition between modes. It includes all rigid-body dynamics of the spacecraft and the two test masses (three translations and three rotations for the spacecraft and each of the test masses). Actuation and measurement noise and major disturbance sources acting on the spacecraft and test masses are modeled. A schematic representation of the ST7-DRS configuration on the LISA Pathfinder is shown in Figure 7, where the Sun exposed face of the spacecraft corresponds to the $+Z$ direction. The location and the orientation of the two test masses can be arbitrarily assigned. The nominal position vectors for the two test masses and their respective housings are chosen as $R_{l}=\left[\begin{array}{llllll}-0.128 & 0 & -0.25\end{array}\right]^{\mathrm{T}} \mathrm{m}$ and $R_{2}=\left[\begin{array}{llll}0.128 & 0 & 0 & -.25\end{array}\right]^{\mathrm{T}} \mathrm{m}$, which means that the sensitive axis is along the $\mathrm{X}$-axis of the spacecraft. Two CMNT clusters, each containing four thrusters, are located on the $\pm \mathrm{X}$ faces to provide thrust capability for attitude and drag-free control. The centers of 
the CMNT clusters are placed at $c_{1}=\left[\begin{array}{lll}-0.9 & 0.125\end{array}\right]^{\mathrm{T}} m$ and $c_{2}=\left[\begin{array}{lll}0.9 & 0 & 0.125\end{array}\right]^{\mathrm{T}} m$, respectively. Each thruster can provide a force that is variable from $5 \mu \mathrm{N}$ to $30 \mu \mathrm{N}$ in $0.1 \mu \mathrm{N}$ increments.

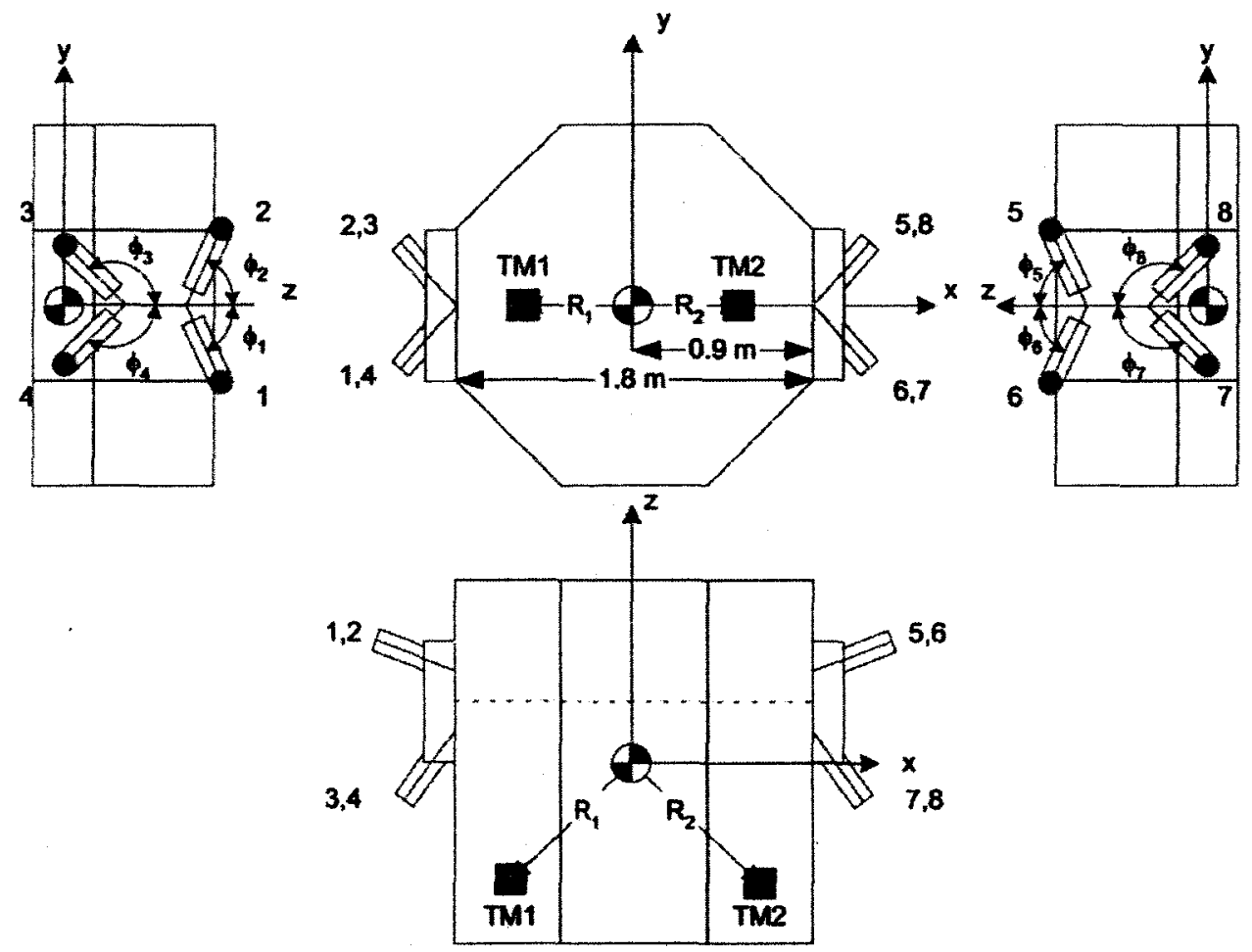

Figure 7. ST7-DRS Configuration on LISA Pathfinder

Disturbances included in this model are solar radiation pressure, acceleration noise on the test mass, capacitive sensing noise, thruster noise, and star tracker noise. The first disturbance modeled is the nominal solar radiation pressure and its variation. The frequency spectrum used for solar radiation flux variations, given in Figure 8, represents a conservative assessment of measured variations. ${ }^{4,5}$ The figure indicates a constant spectrum at frequencies below $0.1 \mathrm{mHz}$, followed by a $1 /$ f roll off. This spectrum also includes the 5 -minute acoustic oscillation (at $3.5 \mathrm{mHz}$ ), and levels off at frequencies above $10 \mathrm{mHz}$.

The second disturbance source modeled was the acceleration noise on the test mass. A number of sources contribute to this acceleration noise, including magnetic and Lorentz forces, thermal disturbances, and cosmic ray impacts. The spectral density function for the test mass acceleration noise is assumed to have: $1 / f^{2}$ roll-off at frequency range of $0.01-0.1 \mathrm{mHz}, 1 / f$ roll-off at $0.1-1 \mathrm{mHz}$, and constant spectral density $3 \times 10^{-14} \mathrm{~m} / \mathrm{s}^{2} / \sqrt{\mathrm{Hz}}$ at frequencies above $1 \mathrm{mHz}$. The linear filter approximation to this frequency spectrum, shown in Figure 9, meets or exceeds all disturbance level requirements and is applied to both test masses in all translational directions.

Capacitive sensing noise was modeled as a colored power spectrum as shown in Figure 10. White-noise models were used to capture thruster and the star tracker noise. The root power spectrum intensity levels were $0.1 \mu \mathrm{N} / \mathrm{JHz}$ for thruster noise and $0.3 \mathrm{mrad} / \mathrm{VHz}$ for star tracker noise. 


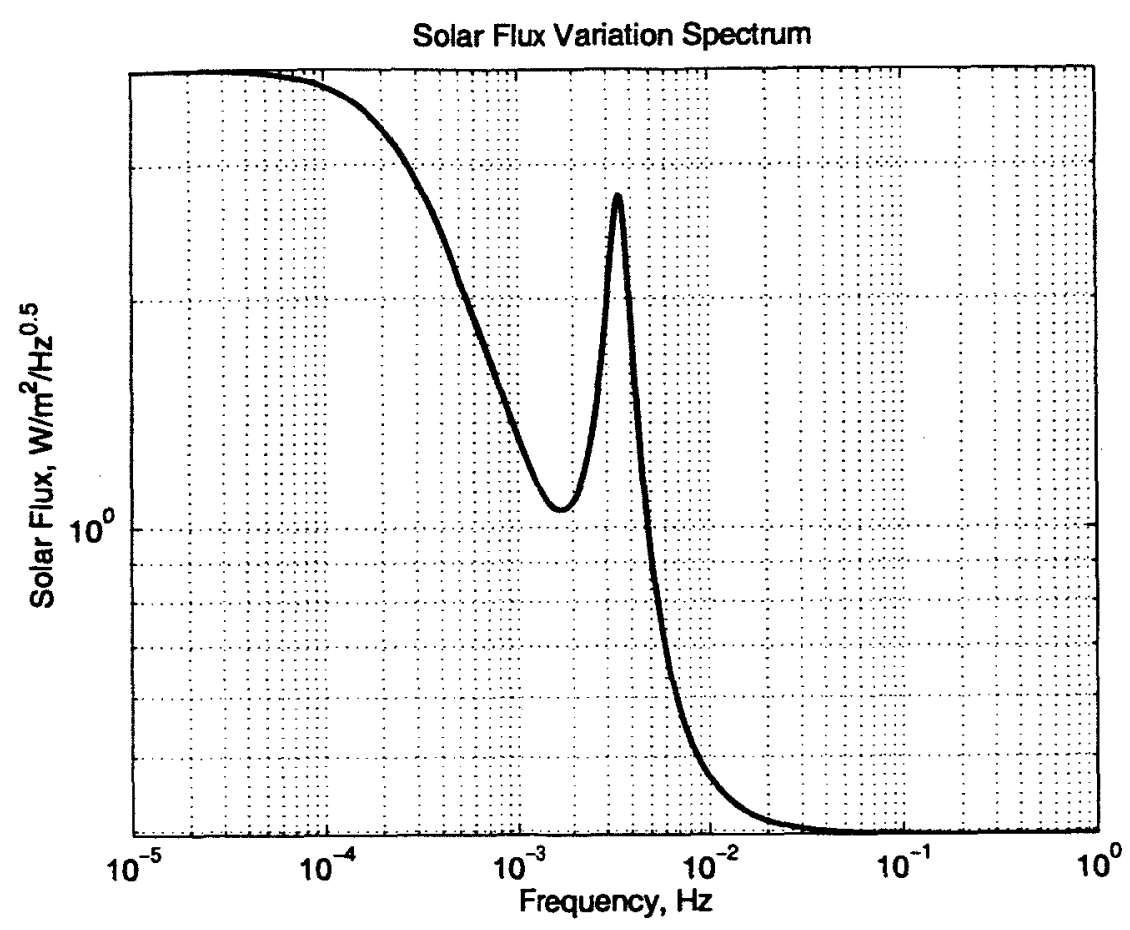

Figure 8. Root Power Spectrum of the Solar Radiation Flax Variation



Figure 9. Root Power Spectrum for Test Mass Acceleration Noise

American Institute of Aeronautics and Astronautics 




Figure 10. Root Power Spectrum of the Capacitive Sensing Noise

\section{Analysis Results and Discussion}

The HiFi model provides the ability to perform an end-to-end analysis of the dynamic performance of the system starting with the AO mode and transitioning all the way to SM mode. A time-domain analysis was performed on the system. The simulation started with the system entering the AO mode with nominal conditions. After 6,000 seconds, the mode is switched to AC mode and is held in that mode for an additional 6,000 seconds. Next, the mode is switched to the DF1 mode and the mean force levels are held. At $t=18,000$ seconds, the mode is switched to the DF2 mode and is held there for 6,000 seconds and at this point, both the force and torque mean values are held. At $\mathrm{t}=\mathbf{2 4 , 0 0 0}$ seconds, the mode is switched over to the SM mode, the force and torque mean values are held, and this mode is held for the duration of the simulation ( $\mathrm{t}=150,000$ seconds). The DRS control modes and transition times are shown in Table 1.

Table 1. DRS Control Sequence

\begin{tabular}{|c|c|c|c|}
\hline Time, Seconds & DCS Mode & RTM Mode & NTM Mode \\
\hline 0 & AO & Accelerometer & Accelerometer \\
\hline 6000 & AC & Accelerometer & Accelerometer \\
\hline 12000 & DF1 & Drag-Free & Accelerometer \\
\hline 18000 & DF2 & Drag-Free & Accelerometer \\
\hline 24000 & SM & Drag-Free & Suspended Drag-Free \\
\hline
\end{tabular}

The results of the analysis are provided in Figure 11-Figure 19. Figure 11 shows the time histories for the spacecraft attitude error. Figure 12 and Figure 13 show the time histories for the GRS position signal (gap) errors for the RTM and the NTM, respectively. Figure 14-Figure 19 show the root PSDs of the gap errors in the SM for both the RTM and NTM. The time history figures indicate that the dynamic performance of the control modes is satisfactory within each mode and during transitions. The transients occurring at the switchover are well contained and understood; hence the transition strategy of direct switchover with control bias carryover is feasible. For example, the first time the spacecraft starts fighting the solar radiation pressure force is at 6,000 seconds when the

8

American Institute of Aeronautics and Astronautics 
DCS mode is switched to AC mode. At this point in time, the DCS and GRS controllers are handing off control authority, which causes the spike in the gap measurement. During the SM mode, the non-reference test mass is in the suspended drag-free mode, meaning that electrostatic suspension loops are soft and are just designed to provide DC and near DC disturbance rejection. Therefore, the apparent steady state error in Figure 13 during SM mode is slowly decaying and despite this offset, the position requirements are still met.

Additional verification of the validity of the transition strategy and the fact that the slowly decaying gap error still meets performance requirements is provided in Figure 14-Figure 19. These figures show the root PSDs of the gap error for both the RTM and NTM based on simulated time history data starting at 25,000 seconds until the end of the simulation. The requirement for the gap error is $10 \mathrm{~nm} / \sqrt{\mathrm{Hz}}$, indicated by the red dashed line, within the frequency band of $1 \mathrm{mHz}$ to $30 \mathrm{mHz}$.

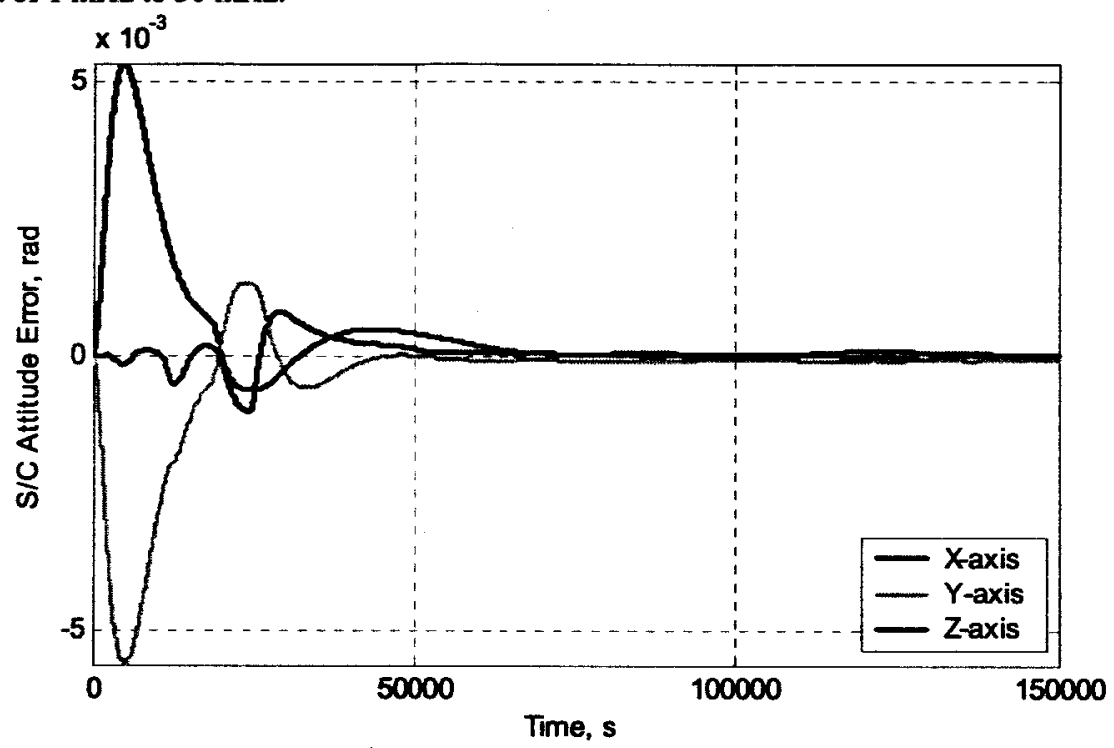

Figure 11. Spacecraft Attitude Error Time History Results



Figure 12. Reference Test Mass Gap Error Time History Results

9

American Institute of Aeronautics and Astronautics 


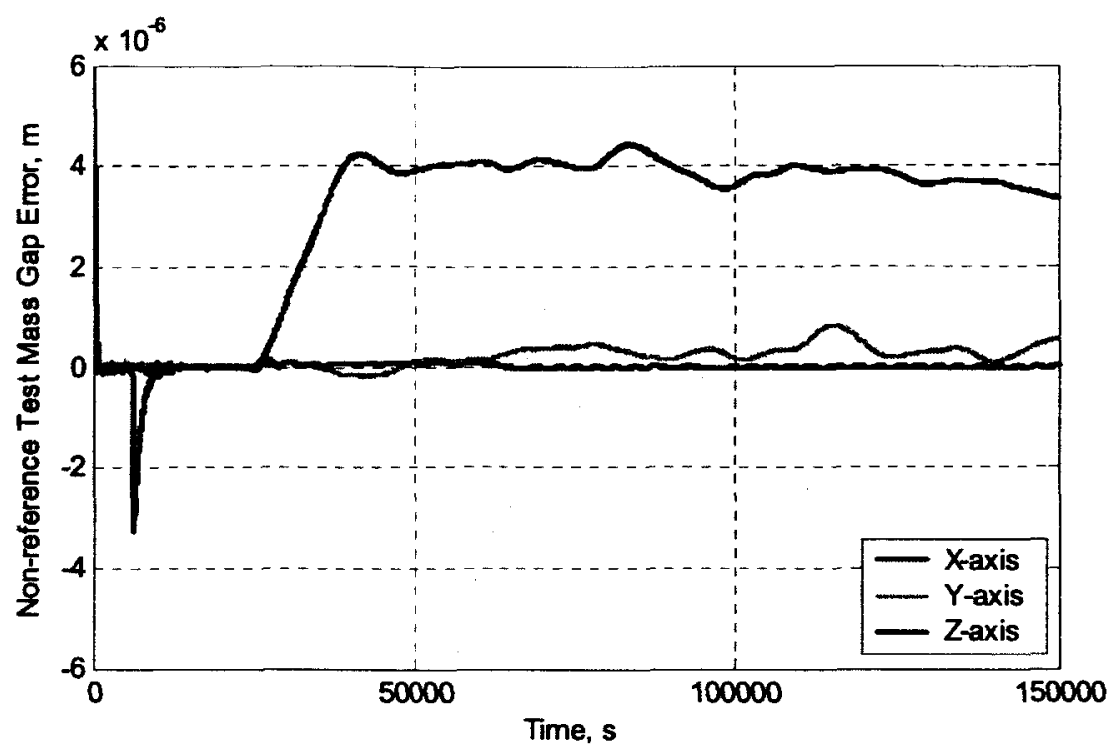

Figure 13. Non-Reference Test Mass Gap Error Time History Results

\section{Concluding Remarks}

Designs for the control modes of the ST7 Disturbance Reduction System have been completed. There are five primary control modes in the current spacecraft control strategy. A mode transition strategy has been presented wherein control is switched directly from one mode to another. However, control effort biases are carried over in between modes to reduce the initial transients at handover. A high-fidelity model of the system has been developed and was used to verify the performance of the control modes and the efficacy of the proposed transition strategy. Both time-domain and frequency-domain analyses indicate that the control modes do meet the DRS requirements, particularly those of the Science Mode. The results indicate that the proposed transition strategy is successful in reducing transients to acceptable levels and that the control system meets its performance requirements.

\section{References}

'Keiser, G. M., Buchman, S., Byer, R. L., Folkner, W. M., Hruby, V., and Gamero-Castaño, M., "Disturbance Reduction System for Testing Technology for Drag-Free Operation," SPIE Paper 4856-02, Astronomical Telescopes and Instrumentation Conference, Waikoloa, Hawaii, USA, August 2002.

${ }^{2}$ Maghami, P. G., Hsu, O. C., Markley, F. L., and Houghton, M. B., "Disturbance Reduction Control Design for the ST7 Flight Validation Experiment," Proceedings of the 2003 Flight Mechanics Symposium, Greenbelt, Maryland.

${ }^{3}$ F. L. Markley, P. G. Maghami, M. B. Houghton, and O. C. Hsu, "18-Degree-of-Freedom Controller Design for the ST7 Disturbance Reduction", Proceedings of the AAS/AIAA Astrodynamics Specialists Conf., Big Sky, MT, August 2003.

${ }^{4}$ Maghami, P. G., Hsu, O. C., Markley, F. L., O'Donnell, J.R., "Control Modes of the ST7 Disturbance Reduction System Flight Validation Experiment, SPIE Paper 5528A-17, International Symposium on Optical Science and Technology (SPIE's 49th Annual Meeting), Denver, Colorado, USA, August 2004.

${ }^{5} \mathrm{~J}$. Pap, et. al., "Variation in Total Solar and Spectral Irradiance as Measured by the VIRGO Experiment on SOHO", Adv. Space Res., 24:215-224, 1999.

GLISA: Laser Interferometer Space Antenna, System and Technology Status Report, ESA-SCI (2000) 11, July 2000. 


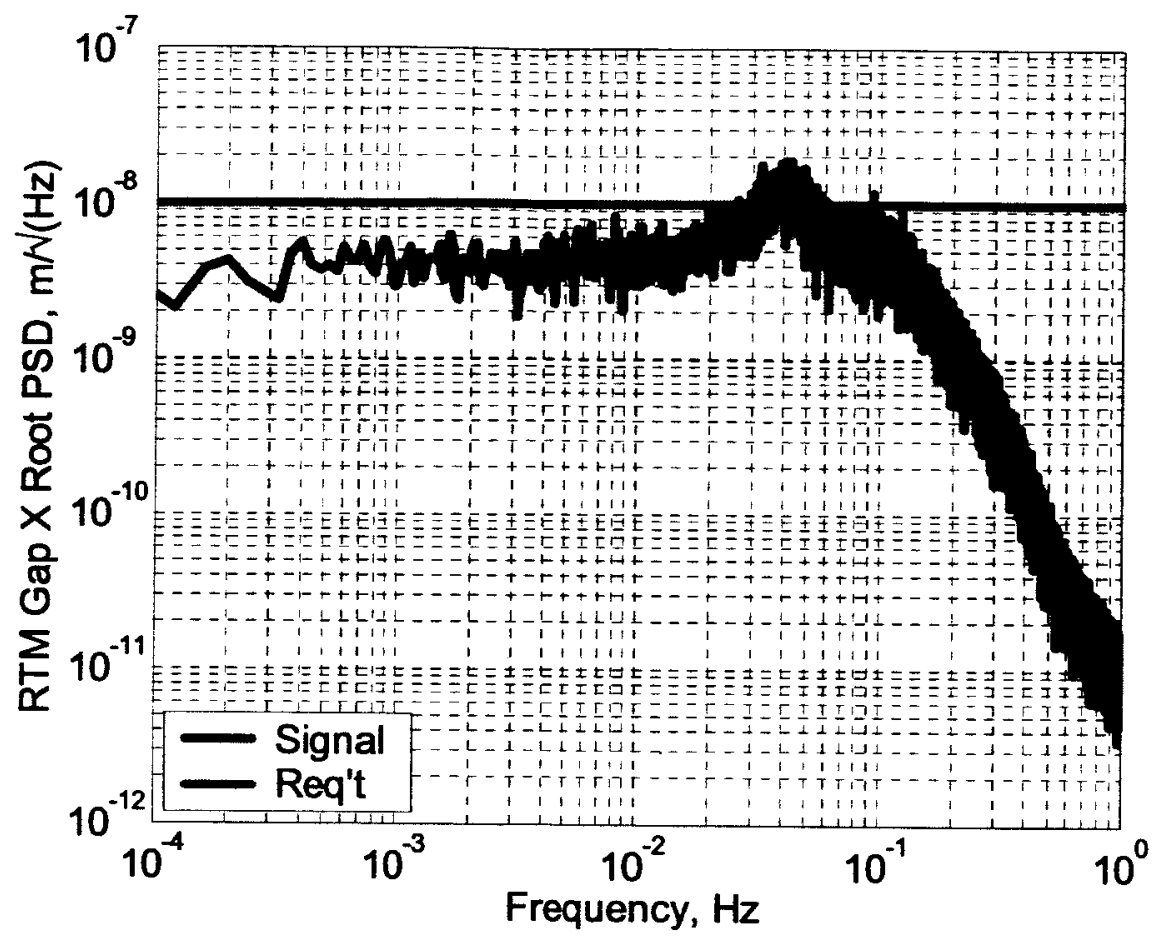

Figure 14. Root PSD for RTM Gap $X$ in SM

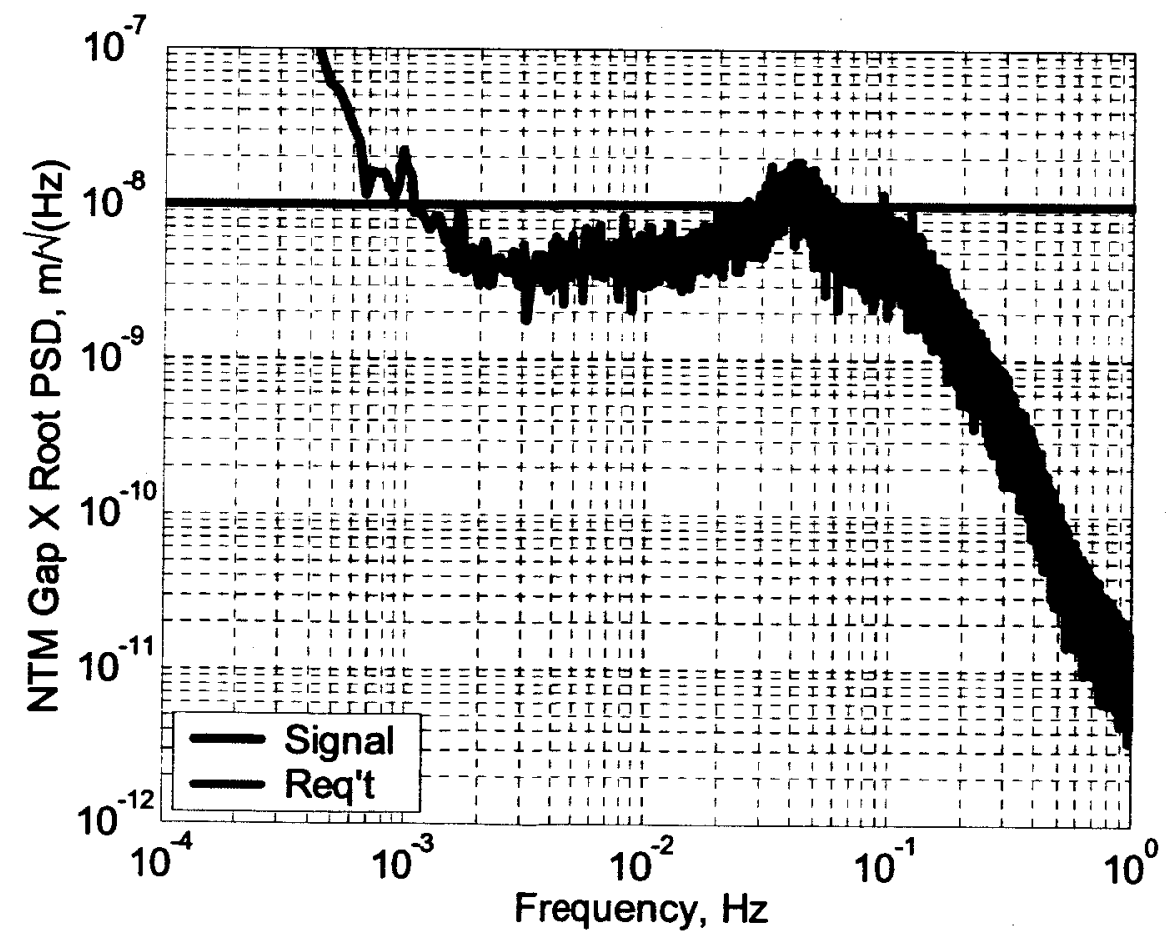

Figure 15. Root PSD for NTM Gap X in SM

American Institute of Aeronautics and Astronautics 


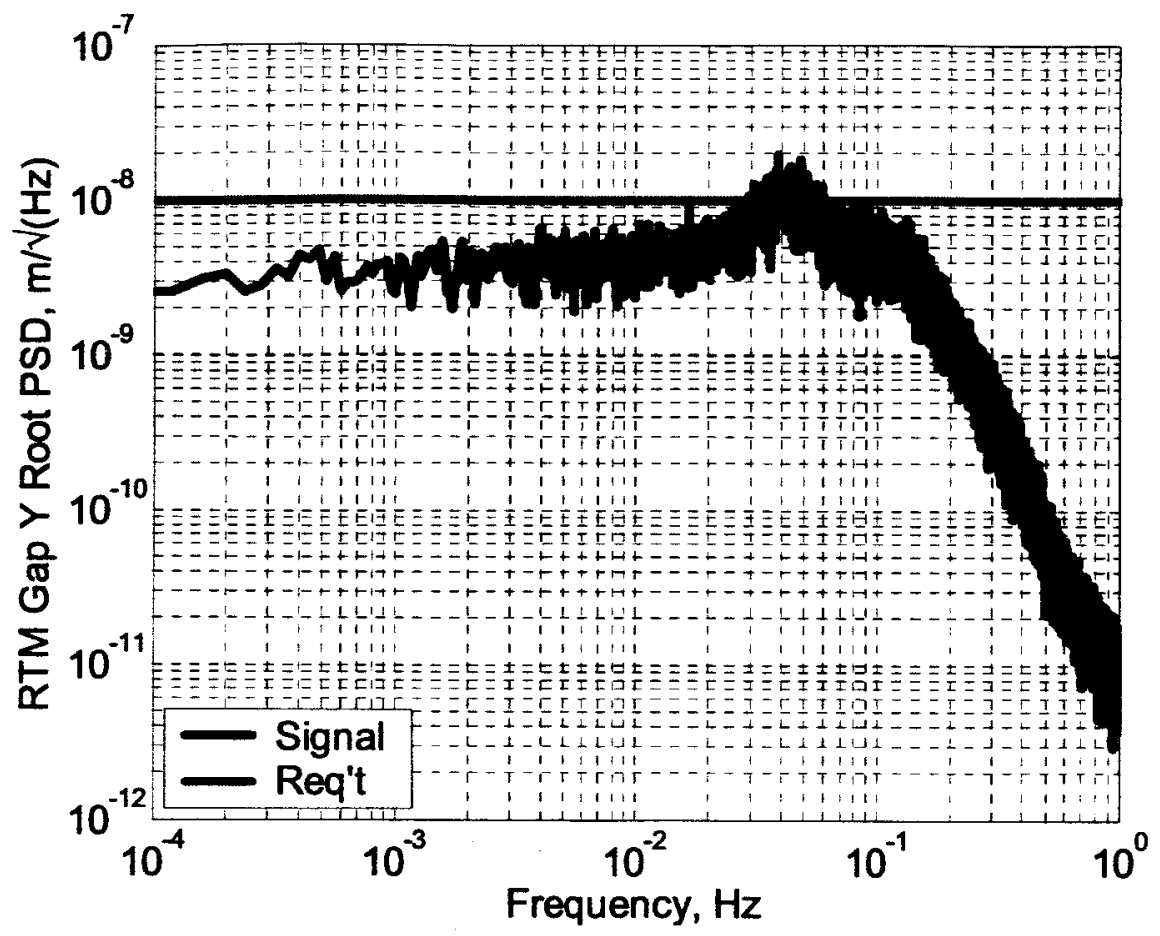

Figure 16. Root PSD for RTM Gap $Y$ in SM

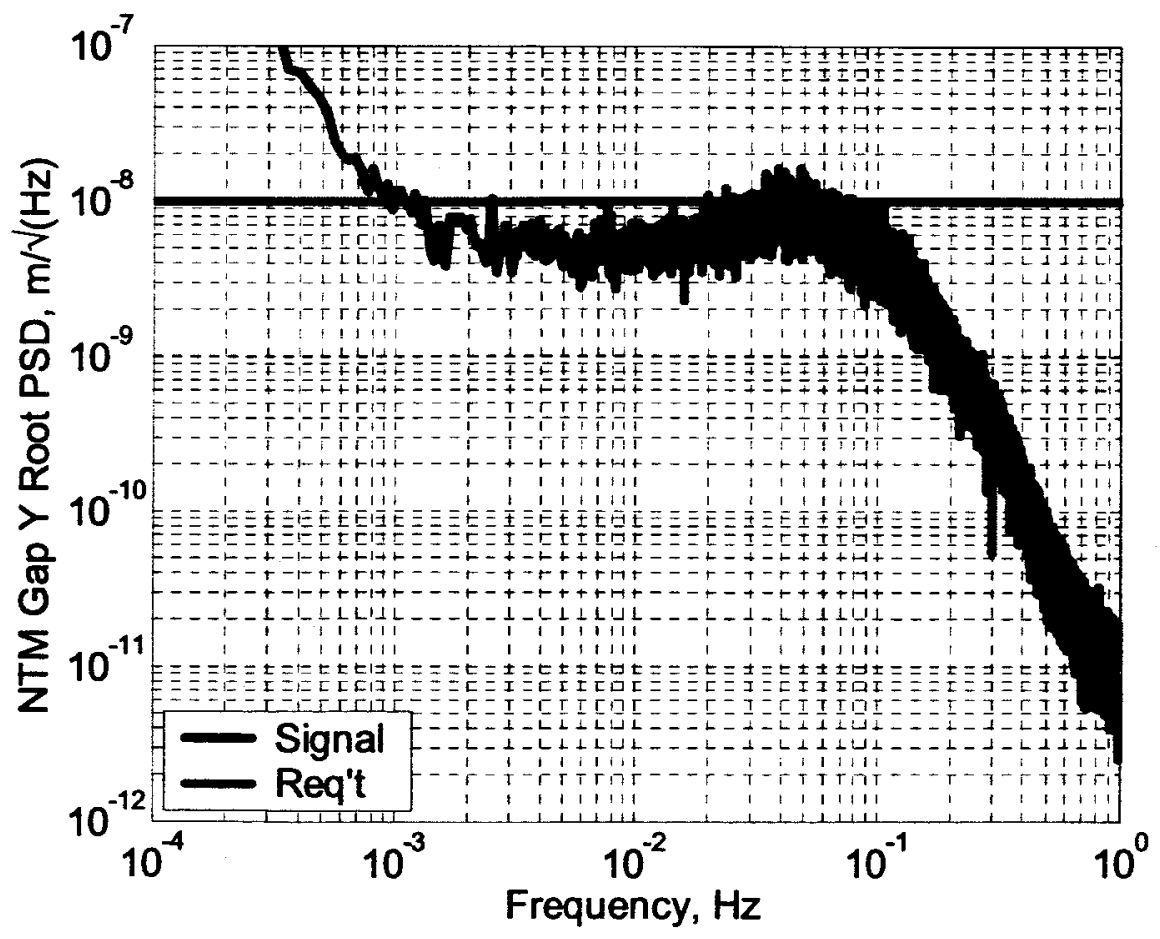

Figure 17. Root PSD for NTM Gap Y in SM

12

American Institute of Aeronautics and Astronautics 


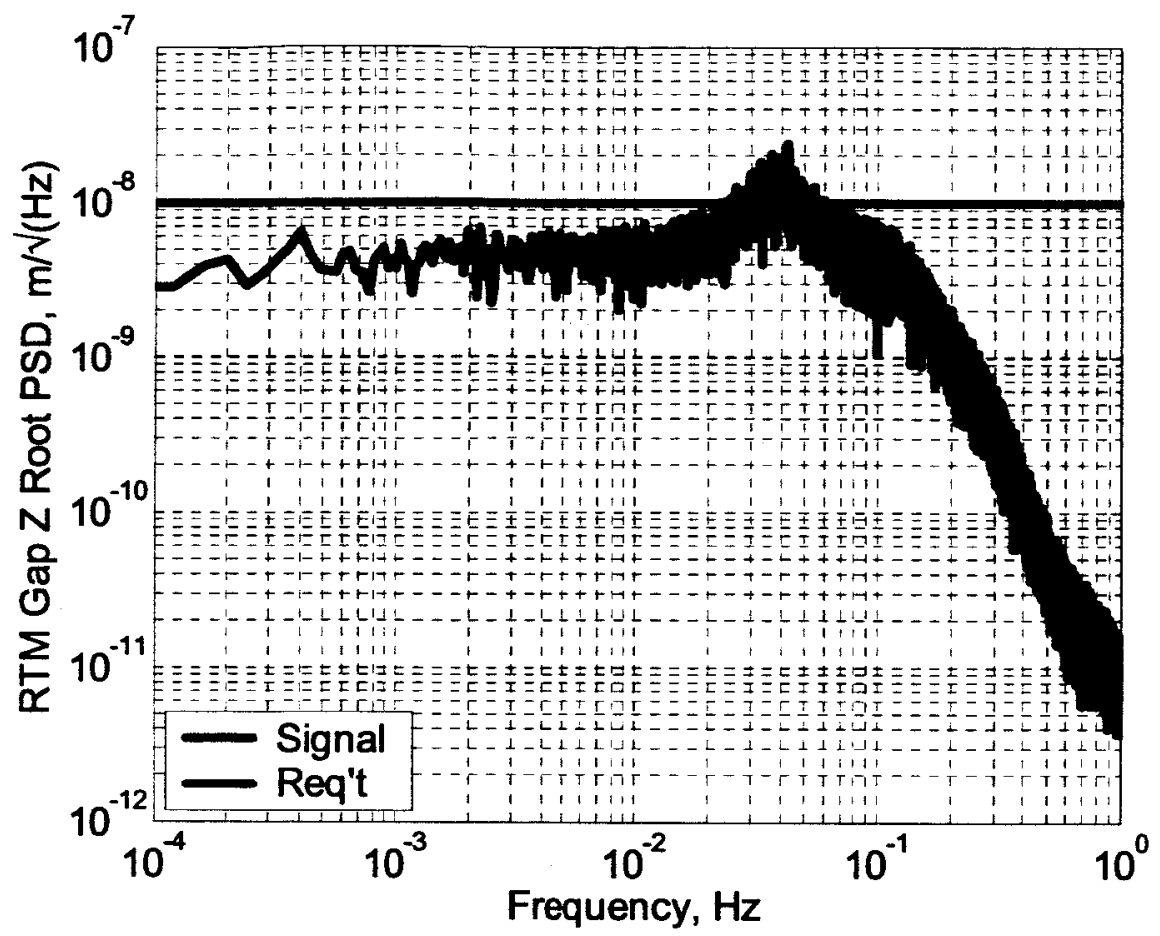

Figure 18. Root PSD for RTM Gap $Z$ in SM

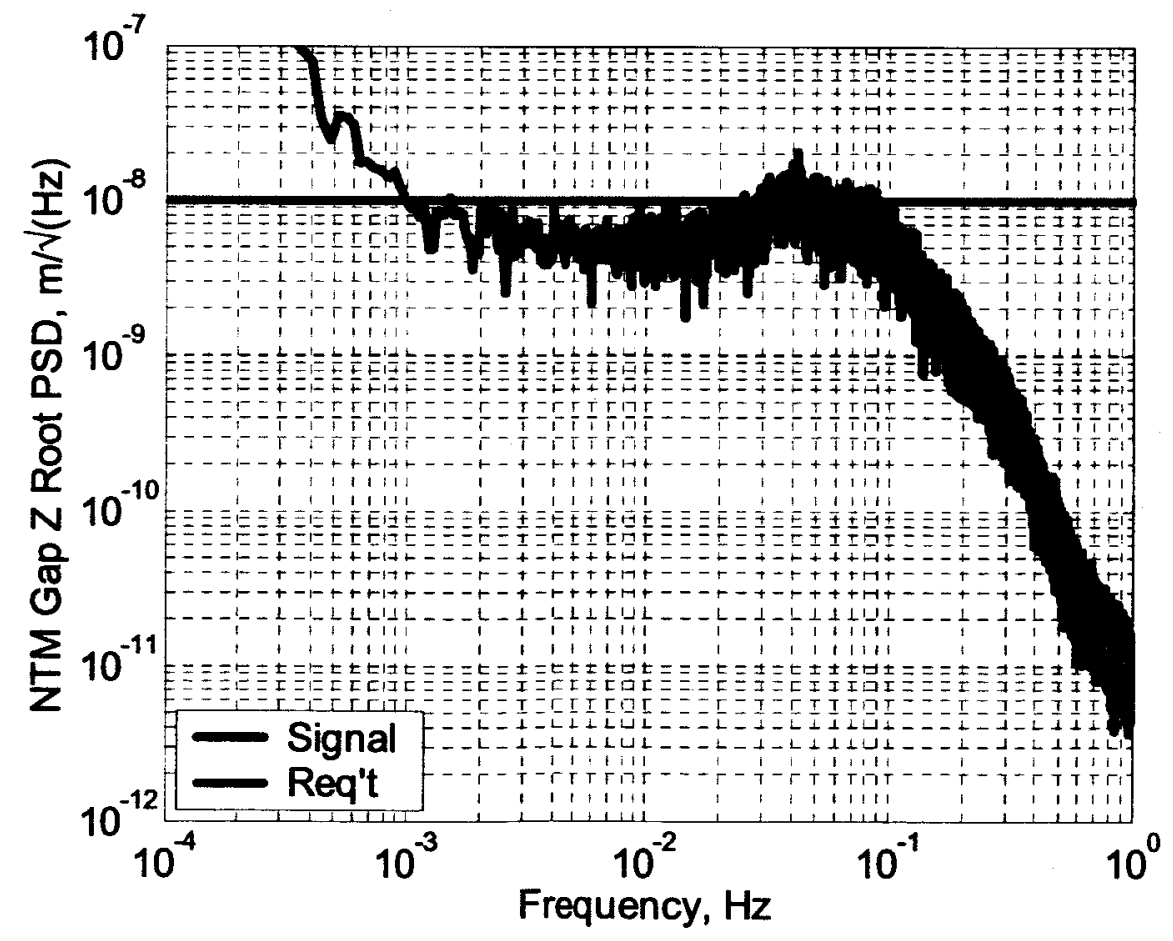

Figure 19. Root PSD for NTM Gap Z in SM

13

American Institute of Aeronautics and Astronautics 